INVESTIGACIONES

EN HISTORIA DE LA

SALUD PÚBLICA

Rev Chil Salud Pública 2013;

Vol 17 (3): 265-274

\section{EL PASADO QUÍMICO-FARMACÉUTICO \\ DE LA REGIÓN DE VALPARAÍSO}

\author{
THE HISTORY OF THE PHARMACY PROFESSION IN \\ the Valparaíso Region
}

\title{
RESUMEN
}

Indagar en el desarrollo bistórico de una profesión es siempre un trabajo interesante, y para el caso de la profesión químico farmacéutica lo es aún más dada su connotación de salud pública y el componente social que tiene. Esta profesión se caracteriza por estar directamente involucrada con la sociedad, y por ende fuertemente ligada al desarrollo de la población. En nuestro país, la expansión de la farmacia, y de la industria farmacéutica, se vio muy favorecida por la llegada de inmigrantes europeos, principalmente alemanes e italianos, quienes contribuyeron al desarrollo de la profesión. Es por este motivo que el presente trabajo de revisión bibliográfica, se centra en el crecimiento de la farmacia en Valparaíso, considerando su carácter de puerto principal del país y de la costa oeste del Pacífico sur, lo que permitió un enriquecedor intercambio comercial y cultural.

Este desarrollo farmacéutico se ha mantenido hasta boy en la región, y a pesar de su importancia para la comunidad de Valparaíso, su pasado ha sido muy poco explorado y reconocido.

\section{INTRODUCCIÓN}

El puerto de Valparaíso nació destinado a convertirse a lo largo de la historia en centro de intercambio comercial y cultural. Este hecho se experimentó con mayor fuerza que en otros puertos del país dada su cercanía

Claudio Badilla Agencia Nacional de Medicamentos Instituto de Salud Pública de Chile cbadilla@ispch.cl con la capital. Su estratégica ubicación lo transformó en sitio de llegada y asentamiento definitivo para muchos inmigrantes, sobre todo de Europa, quienes, buscando suerte en la ciudad, se establecieron con casas comerciales. Es de hecho gracias a ellos que se conocieron los primeros "establecimientos farmacéuticos" que existieron en el puerto. 
Todo ha girado en torno a Valparaíso y su progreso económico y comercial trajo la creación de numerosas industrias. Sin embargo, la ciudad -y la región en general- ha sufrido enormes desastres naturales, lo que ha significado prácticamente tener que rehacerla una y otra vez. Numerosas empresas se han visto obligadas a migrar hacia otras regiones, principalmente a la Metropolitana de Santiago, pero aun así todavía quedan en la zona industrias químico-farmacéuticas, químicas y de alimentos. Llama la atención sí, que las antiguas y ya desaparecidas industrias farmacéuticas de la quinta región de Valparaíso, que fueron de gran relevancia para la población, no sean recordadas entre sus habitantes. $\mathrm{Mu}$ chos de los laboratorios farmacéuticos para los que se recopilaron antecedentes, son casi desconocidos para la comunidad porteña, y es precisamente de este hecho que surge la iniciativa de realizar esta investigación.

Desde sus inicios hasta hoy, el crecimiento de la profesión farmacéutica en la región no se ha detenido. Así lo confirma la creación de la Facultad de Farmacia de la Universidad de Valparaíso, que ha sido uno de los factores relevantes para el continuo progreso de la profesión.

Para este trabajo se realizó una revisión bibliográfica de revistas de las organizaciones químicas farmacéuticas a lo largo de la historia del país, desde los primeros años del siglo XX hasta la década de 1980, además de publicaciones acerca de la historia social y comercial del puerto de Valparaíso. Las fuentes bibliográficas pertenecen a la Biblioteca $\mathrm{Na}$ cional de Chile y a archivos personales.

\section{LOS PRIMEROS AÑOS DE LA REPÚBLICA}

A partir de 1852 Valparaíso cuenta con un servicio de agua potable (1). Mucho antes de la existencia de estas obras que contribuyeron a la salubridad de la ciudad, ya había antecedentes de establecimientos químico-farmacéuticos en ella.

Uno de estos antecedentes lo constituye una de las primeras apreciaciones de cómo era una botica en Valparaíso, de acuerdo a una descripción hecha por la viajera y escritora inglesa Mary Graham en 1822, en donde describe un espacio bastante desordenado y falto de pulcritud.

De vuelta de mi excursión por las tiendas, pasé donde el boticario (porque no hay aquí más que uno) para comprar un poco de azul, que con gran sorpresa mía supe que ahí podía procurarse. Su vista me hacía pensar en un boticario del siglo XIV, porque es de un aspecto mucho más anticuado que los que he visto en Francia y en Italia. Tiene cierto gusto por la historia natural, de manera que a más de sus tarros de medicinas pasadas de moda, rotulados con signos cabalísticos, confusamente revueltos con paquetes de medicinas de patente de Londres, hierbas secas y sucias vasijas de greda, hay cabezas de pescado y cueros de serpiente [...] En un rincón se ve un gran cóndor arrancando la carne de los huesos de un cordero; en otro, un monstruoso carnero que tiene una pata de más que le nace en la frente. (2)

Durante el siglo XIX aparecieron muchas enfermedades a consecuencia de los problemas de tipo sanitario existentes en la ciudad. A consecuencia de ello una de las medidas que adoptó la autoridad a modo de protección de la salud de sus habitantes fue la creación, en 1892, del Laboratorio Químico Municipal, destinado a regular la calidad y condiciones de los productos alimenticios que se consumían (3). Se señala que la ciudad de Valparaíso contaba con un laboratorio químico antes que Santiago y que uno de los primeros fundados en el puerto fue el del farmacéutico Emilio Eisele (4).

El desarrollo de estos establecimientos se vio favorecido por la condición de puerto comercial más importante de la costa del Pacífico, debido al descubrimiento de oro en California que, sin duda, repercutió positivamente en la marcha ascendente del primer puerto de Chile (3). Su época dorada se produjo entre la segunda mitad del siglo XIX y 1914, fecha de inauguración del canal de Panamá, obra que acabó con el auge comercial del puerto, ya que las 


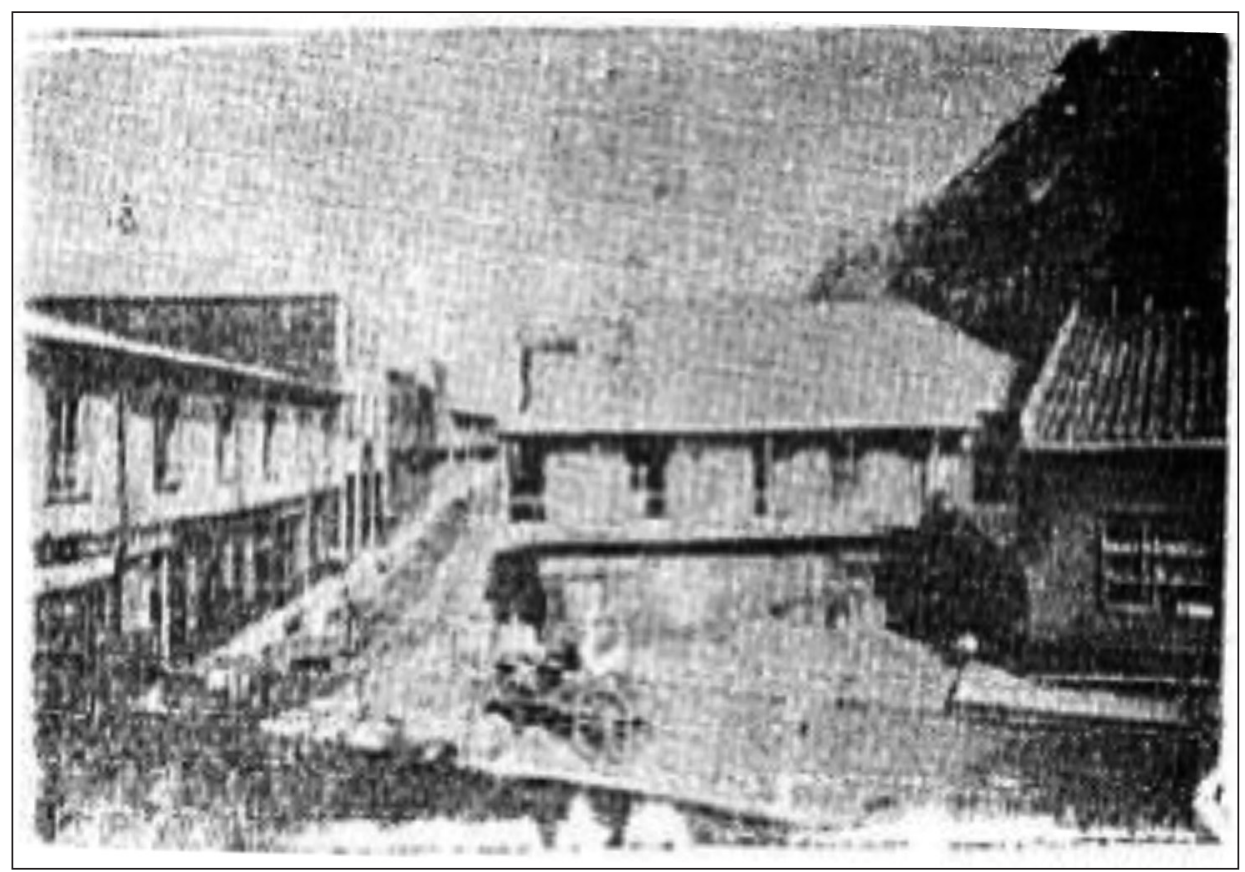

Figura 1. Primera droguería establecida en Valparaíso, en la esquina de la calle del Comercio con la quebrada de San Agustín, en 1837. (1)

embarcaciones comerciales no tenían la necesidad de cruzar el Cabo de Hornos como ruta obligada hacia Estados Unidos. Esto provocó que Valparaíso perdiera su condición de puerto de paso en rutas comerciales y punto de llegada de muchos inmigrantes que contribuyeron al avance de las iniciativas industriales farmacéuticas, químicas y cosméticas en la región. Un rol sobresaliente cumple en ello la colectividad británica y particularmente la alemana, de cuyo predominio en el manejo de las farmacias y laboratorios químicos puede dar fe la propiedad mayoritaria de las boticas existentes a mediados del siglo XIX (cinco de las diez que había). No era extraño que dueños de boticas de otras nacionalidades buscaran farmacéuticos alemanes, para aprovechar la fama que estos tenían en la especialidad (3).

Tenemos también el caso del inmigrante italiano Antonio Puccio, quien establece su botica y droguería en 1837, la que se ubicaba en la calle Del Comercio, hoy calle Prat (actual edificio Interoceánica). Con posterioridad, esta botica se convertiría en la primera industria farmacéutica de Chile, con el nom- bre "Farmoquímica del Pacífico". Durante su existencia, la empresa, con laboratorios en Valparaíso y Llay-Llay, tuvo una fuerte presencia de inmigrantes alemanes, quienes aportaron a su desarrollo entre la segunda mitad del siglo XIX y la primera del XX.

Además de ser pionero, este es quizá el desarrollo industrial farmacéutico más importante ocurrido en Valparaíso, razón por la que se le dará especial énfasis aquí. Su relevancia de hito histórico quedó plasmada en el trabajo desarrollado por María Alliende Edwards en 1987 titulado Los inicios de la industria químico-farmacéutica en Chile: un caso particular: historia de Farmoquímica del Pacífico S.A. (1834-1987), editado por la Academia de Ciencias Farmacéuticas de Chile.

Farmoquímica del Pacífico se caracterizó por ser una empresa que nació y se desarrolló gracias a los inmigrantes, y que con el tiempo pasó de manos de unos a otros, hasta hacerla crecer y posicionarse en el mercado farmacéutico nacional, junto con la creación de empresas menores bajo su alero, que permanecen hasta el día de hoy. 


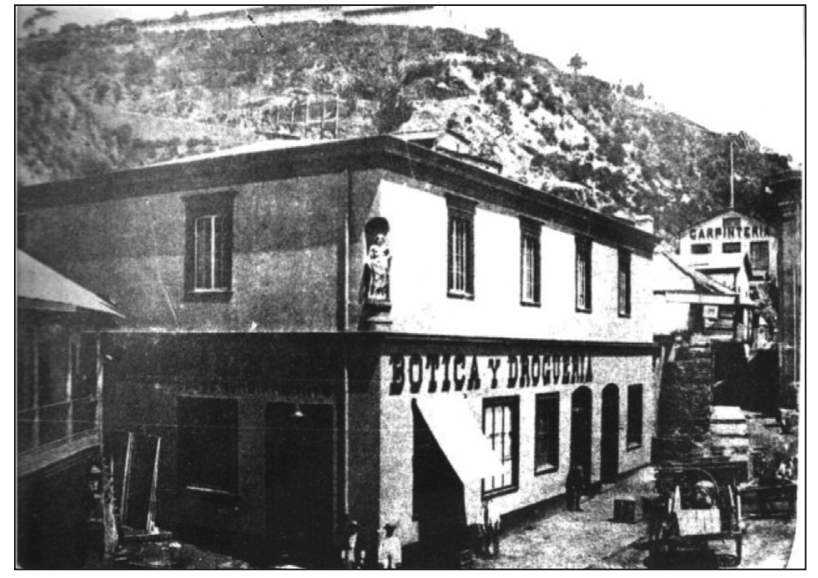

Figura 2. Fotografía de 1868, en donde se ve a la sucesora de la empresa fundada por Antonio Puccio (Mongiardini y Cia.), en el mismo lugar de la fotografía anterior (actual sector de la plaza de justicia), y es la antecesora de Daube y Cía., posterior Farmo-Química del Pacífico (5).

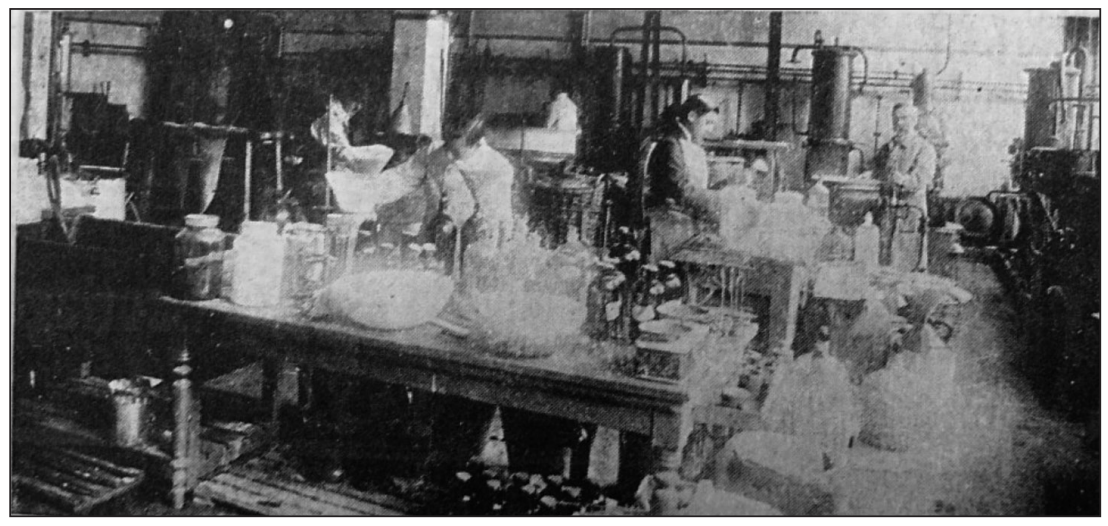

Figura 3. Interior del laboratorio químico-farmacéutico de Daube y Cia.

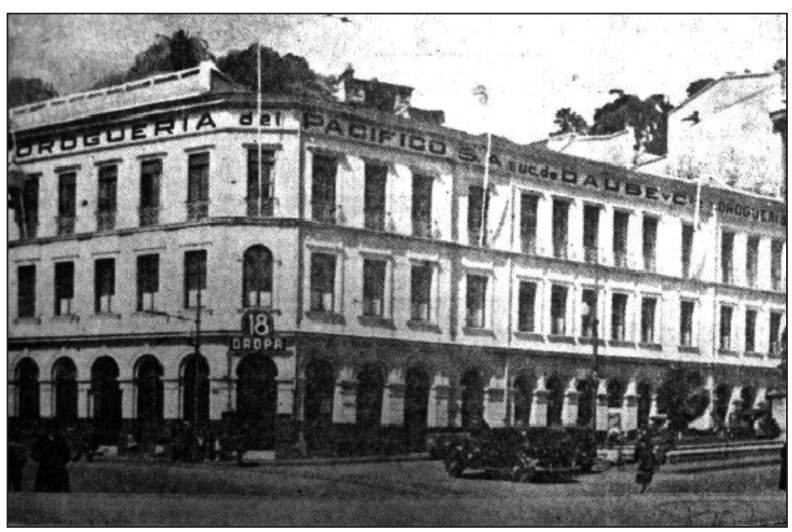

Figura 4. Edificio de la Droguería del Pacífico, sector de plaza de justicia y plaza Sotomayor en Valparaíso. 
La empresa fue cambiando de razón social en numerosas ocasiones, desde la firma Mongiardini, pasando por Daube y Cía, Laboratorios 18, Droguería del Pacífico DROPA hasta llegar finalmente a Farmo-Química del Pacífico.

La empresa paulatinamente fue trasladando sus operaciones desde Valparaíso hacia el interior de la región, específicamente a la localidad de Llay-Llay, donde se construyó la planta farmacéutica.

Allí la empresa recibía la visita de estudiantes de farmacia, para que se interiorizaran en los procedimientos e instalaciones propios de las operaciones farmacéuticas.

Con el tiempo, se trasladó a la ciudad de Santiago, cuyas instalaciones se ubicaban en la calle Santo Domingo, en el centro de la ciudad.

\section{EL SIGLO XX}

Valparaíso ha sido una ciudad golpeada por los desastres o embates de la naturaleza, pero aun con tragedias tan devastadoras como el gran terremoto del 16 de agosto de 1906, la sociedad porteña supo llevar adelante su desarrollo.

En Valparaíso no solamente hubo desarrollo de laboratorios químico-farmacéuticos, sino de muchas fábricas de productos químicos, alimenticios y de jabones.

Entre los casi olvidados laboratorios que existieron en la región están Laboratorio Londres de H.V. Prentice, con productos premiados en exposiciones nacionales e internacionales; Laboratorio Bioquímico de Valparaíso, que destacaba por la buena base científica de sus formulaciones y por la elaboración de inyectables; Laboratorio Sthenogen, Laboratorio Sanax, con productos cosméticos, farmacéuticos y alimentos fosfatados; Laboratorio Munier; los Laboratorios Albión, Galeno, la Droguería Belga ubicada en el cerro Barón, y la Droguería Británica de propiedad del químico farmacéutico M.O. Coghlan. En tanto, en la producción de cosméticos destacaba el Laboratorio Riegel.

En Viña del Mar existió el Laboratorio Sanino de productos químicos y farmacéuticos; el Laboratorio de Annibali y Bórquez; en Recreo estuvo el laboratorio del químico farmacéutico Teodoro Boye. Este último, junto con el Laboratorio Munier, ayudaría posteriormente a formar los laboratorios de la Escuela de Química y Farmacia de la Universidad de Valparaíso. En la ciudad de La Calera existió, por su parte, el Laboratorio Salus.
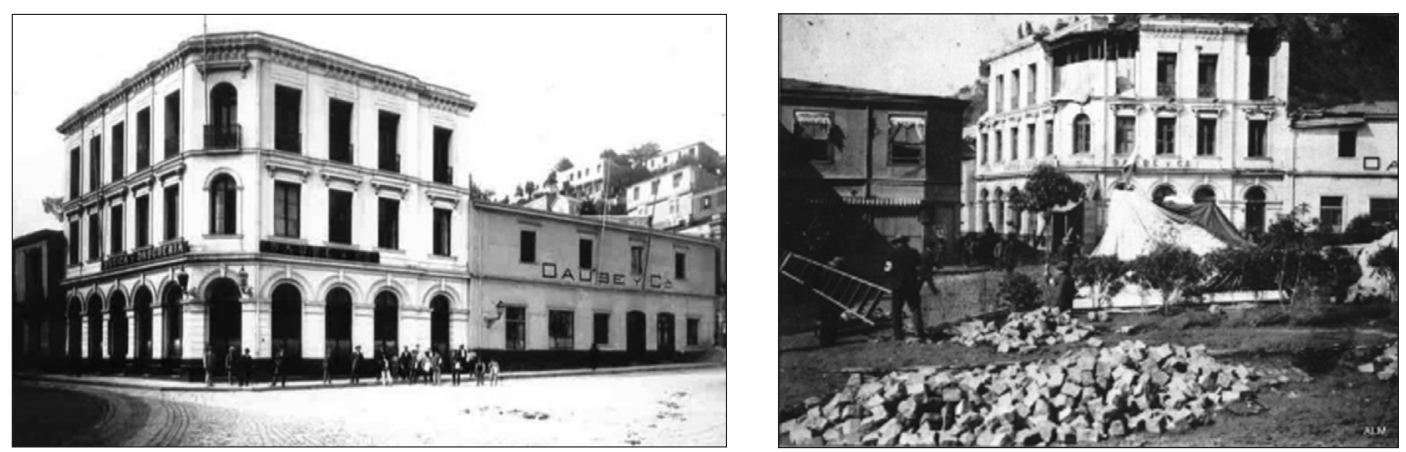

Figura 5. El destruido edificio de Daube y Cia. a consecuencia del terremoto del 16 de agosto de 1906. Esta imagen aparece en una publicación de la Academia de Ciencias Farmacéuticas de Chile, relacionada con la historia de la farmacia en Valparaíso, y en ella se indica que el establecimiento funcionó de forma provisoria en esas “carpas o tiendas” que se ven en la imagen. 


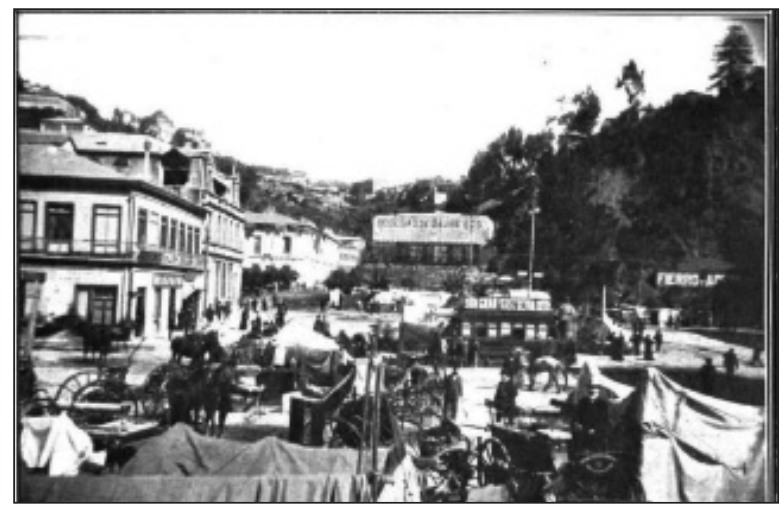

Figura 6. Sector de plaza Sotomayor en Valparaíso luego del terremoto de 1906. Al fondo se ven las bodegas de Daube y Cia.

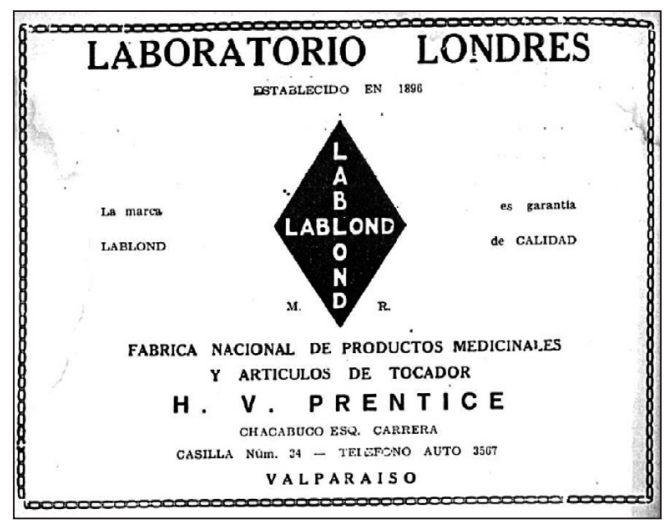

Figura 7. Anuncio publicitario de Laboratorio Londres de Valparaíso (6).

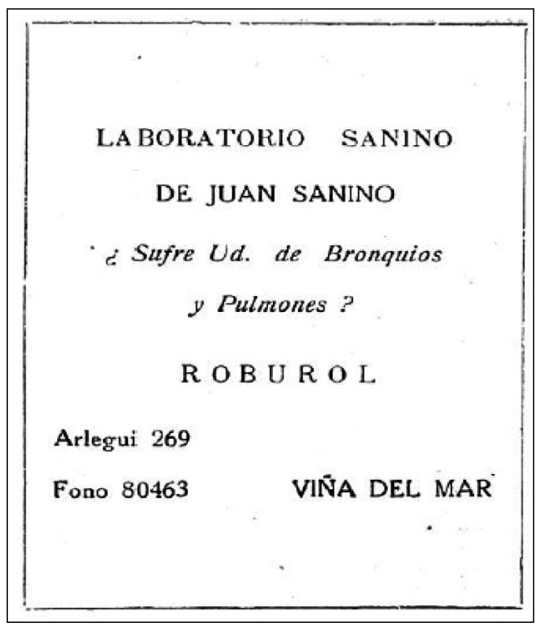

Figura 8. Anuncio publicitario de Laboratorio Sanino de Viña del Mar (1).
Estos datos corroboran que la región de Valparaíso fue un destacado polo de producción químico-farmacéutica.

Muchas de las industrias químico-farmacéuticas, así como también laboratorios internacionales, participaron en la exposición de industrias y comercio de Valparaíso en 1936. Los laboratorios participantes de la región de Valparaíso fueron: Droguería del Pacífico, Laboratorio de Annibali y Bórquez de Viña del Mar, Farmacia El Mercurio de Valparaíso, Farmacia Unión de Viña del Mar, Laboratorio Valin de Valparaíso. Dentro de los stands destacados en la exposición estaba el de "Laboratorio Albión", de propiedad del farmacéutico porteño señor M.O. Coghlan, quien exhibió principalmente preparaciones de tocador y algunas de uso medicinal externo. Debe mencionarse también al Laboratorio Bioquímico de Valparaíso, que en un voluminoso stand presentó sus productos inyectables y cuya producción se distinguió por las fórmulas científicas que utilizara en sus preparados (7), además de destacarse por su participación en publicaciones científicas tales como Contribución al estudio del tratamiento del raquitismo por el golpe vitamínico, en conjunto con personal del hospital Enrique Deformes de Valparaíso en 1941. (8)

De dicha exposición quedó de manifiesto la buena impresión ofrecida por los laboratorios y el progreso experimentado por la industria farmacéutica en el país (7). 


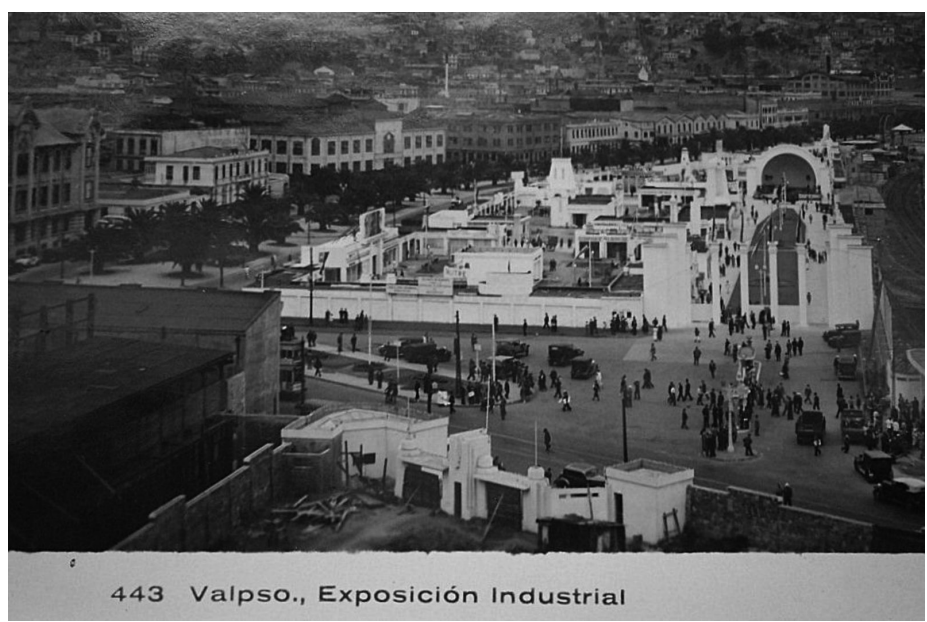

Figura 9. Exposición industrial de 1936 en Valparaíso, sector de El Almendral.

En publicaciones como las revistas del colegio químico farmacéutico, los almanaques de Daube (posteriormente Laboratorios 18 y Farmoquímica del Pacífico), y la revista Sucesos, durante la primera mitad del siglo XX (específicamente entre 1915 y 1950), se publicitaban una gran cantidad de productos farmacéuticos que abarcaban numerosas formas farmacéuticas y productos cosméticos, como los señalados a continuación:

\section{Productos Farmo-Química del Pacífico:(9)}

- Sal de fruta Frutalin 18

- Pasta Perlam 18

- Agua de colonia Juanita

- Petrol champú (con petróleo sin olor)

- Pinomarol 18 (para la bronquitis crónica), jarabe de pino marino.

\section{Productos Laboratorio Munier:(10)}

- Nodesset: Antialérgico-desensibilizante inespecífico-inyectable.

- Activogeno: Reconstituyente-hematopoyético-elíxir.

- Dolanyl: Galactógeno y mineralizanteJarabe.

\section{Productos Laboratorio Bioquímico de Val- paraíso:(10)}

- Ossagen: Vitaminas A, B, D y E, extracto hepático, sales de calcio, magnesio y hierro, en gránulos.

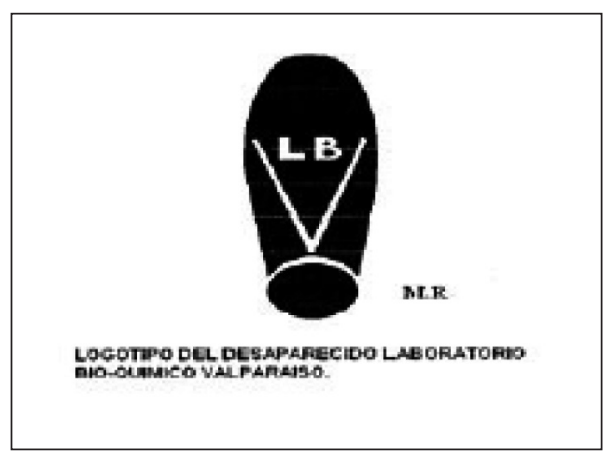

Figura 10. Logotipo Laboratorio Bioquímico de Valparaíso.

Productos Laboratorio Londres H.V. Prentice-Valparaíso:(11)

- Fenaloína Lablond M.R: Píldoras contra el estreñimiento en base a fenolftaleína y aloína.

\section{Productos Laboratorio Sanino:(11)}

- Normalina, para insomnios.

Fábrica de envases S.A. Valparaíso:(12)

- Especialistas en envases para productos de belleza y farmacéuticos.

Durante el siglo XX tenemos la aparición de farmacias que posteriormente se transformarían en grandes empresas con presencia a nivel nacional e internacional. Tales son los 


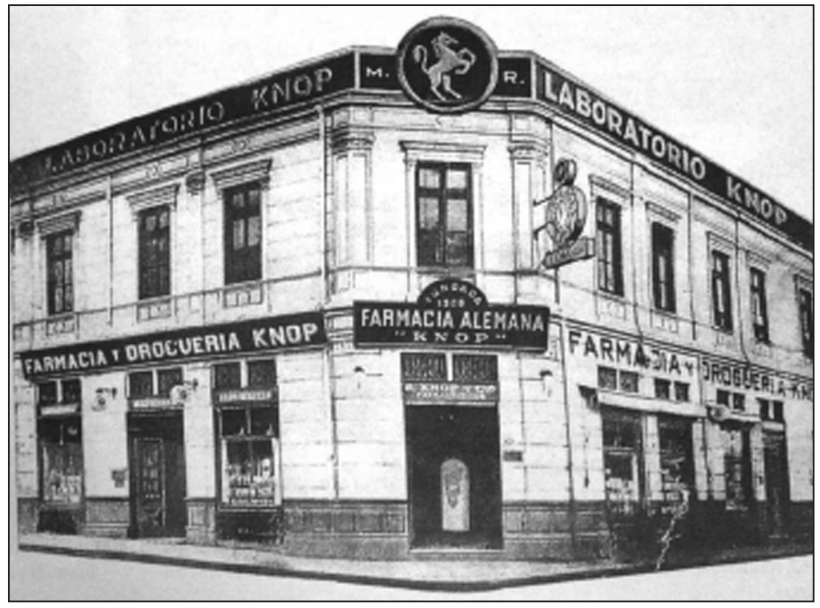

Figura 11. El primer local de farmacias Knop, en el sector de plaza Echaurren en pleno barrio puerto de Valparaíso.

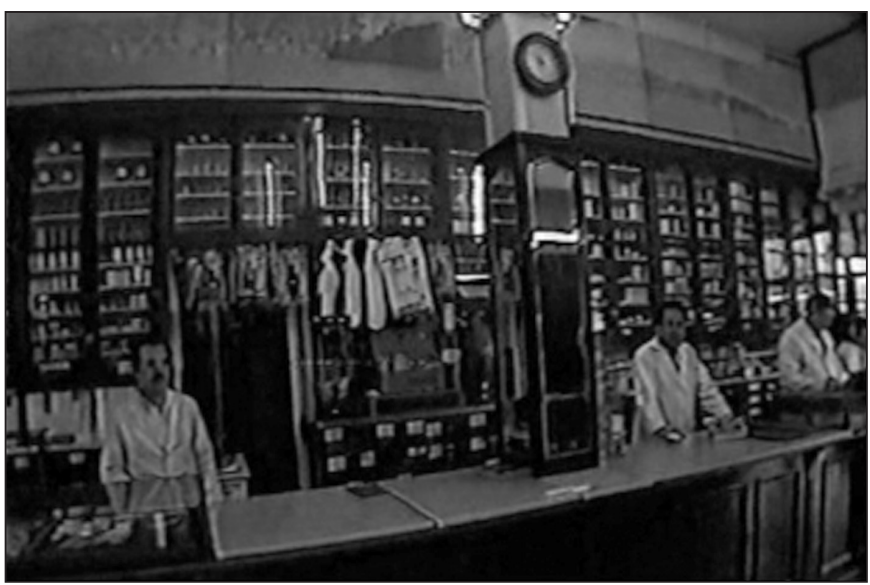

Figura 12. Interior de la antigua y ya desaparecida Farmacia Knop de la plaza Echaurren de Valparaíso.

casos de Laboratorios Knop y Farmacias Cruz Verde. Recordada es la antigua Farmacia Knop, ubicada frente a la plaza Echaurren en pleno barrio puerto de Valparaíso, empresa que nace en 1931 y siempre se ha orientado a la fabricación de productos homeopáticos y naturales.

Un hito importante en la historia del desarrollo farmacéutico de la región de Valparaíso, fue la creación, en 1972, de la escuela de Química y Farmacia, que con el tiempo se transformaría en la actual Facultad de Farmacia de la Universidad de Valparaíso. El 18 de julio de 1972, se constituyó la Comisión Profesional Provisoria de la Carrera de Química y Farmacia, cuyo presidente fue el jefe de la carrera, doctor Ernesto Fernández Bernardo (13).

Uno de los primeros antecedentes que se tiene del proyecto de crear una escuela de Química y Farmacia aparece en una publicación del Colegio Químico Farmacéutico de 1967 acerca del Instituto de Química de la Universidad de Chile en Valparaíso(13, 14).

Los laboratorios de la Escuela de Química y Farmacia de Valparaíso fueron implemen- 


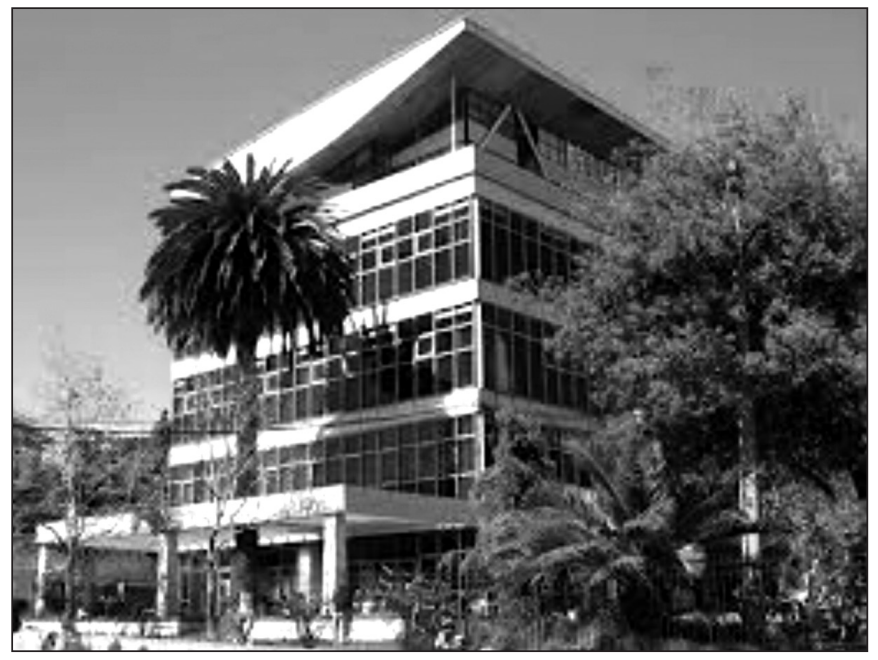

Figura 13. Actual edificio de la Facultad de Farmacia de la Universidad de Valparaíso, la que alberga a las carreras de Química y Farmacia, y Nutrición.

tados gracias a la compra de maquinarias, muebles e instalaciones del laboratorio de producción farmacéutica Munier de propiedad del farmacéutico señor Teodoro Boye, quién además fue uno de los primeros profesores de la cátedra de Tecnología Farmacéutica. $(13,15)$

\section{DISCUSIÓN}

Es innegable que el desarrollo industrial de la profesión químico farmacéutica ha ido de la mano con el desarrollo portuario de Valparaíso. Sin embargo, debemos señalar que esta situación se dio hasta la mitad del siglo $\mathrm{XX}$, debido a que acontecimientos como el terremoto de 1906, la construcción del puerto de San Antonio en 1912 y la apertura del canal de Panamá hicieron que paulatinamente Valparaíso dejara de ser una plaza comercial atractiva y por ende viera disminuida la llegada de inmigrantes y de aporte tecnológico. A lo antes mencionado, se debe agregar que la condiciones de las administraciones portuarias en nuestro país a partir de la segunda mitad del siglo XX, fueron totalmente diferentes a las existentes durante la "época de oro" del puerto de Valparaíso (fines del siglo XIX hasta la primera mitad del XX), dada la estatización de los puertos con la creación de la Empresa Portuaria de Chile, EMPORCHI, en 1961, y su posterior privatización. Sumado a esto, la concentración de las actividades industriales y comerciales en la región Metropolitana de Santiago trajeron consigo una disminución de las iniciativas industriales en Valparaíso, no solo farmacéuticas, sino que en diferentes rubros.

Aun así, como un legado del pasado de desarrollo químico farmacéutico originado desde los primeros años de vida independiente de este país, en la actualidad todavía se mantienen industrias de tipo químico y farmacéuticas en la región, las que, en conjunto con la Facultad de Farmacia de la Universidad de Valparaíso, siguen contribuyendo al desarrollo de la química y farmacia.

\section{REFERENCIAS}

1. Aguirre L. El libro de Valparaíso: Historia-Educación-Cultura-Prensa y Radiodifusión-Acción Social-Comercio industria-Vida Social-Benefactores-Muertos inolvidables-Colectividades extranjeras-Cuerpo consular-Profesionales-Vida obrera-Problemas-Vida porteña a través de los años-Deportes-Turismo-Ciudades de la 
provincia 1536-1946. Valparaíso: Salesiana. 1946.

2. Almanaque 18. Edición 68. Santiago: Farmoquímica del Pacífico S.A. 1987.

3. Estrada B. Valparaíso: Sociedad y economía en el siglo XIX. Valparaíso: Universidad Católica de Valparaíso, Instituto de Historia, Facultad de Filosofía y Educación. 2000.

4. Revista Farmacéutica Chilena. 1902; 1(1).

5. Almanaque 18. Edición 42. Santiago: Farmoquímica del Pacífico S.A. 1961.

6. Órgano oficial de la Asociación Chilena de Química y Farmacia. Editada por la Junta Provincial de Valparaíso. Revista Químico Farmacéutica. 1938-1939.

7. Gran parte de la producción farmacéutica chilena está representada en la exposición de industrias y comercio de Valparaíso. Revista Química Farmacéutica-Valparaíso. 1937; IV(40).

8. Muzzo S, Carreon C, Domínguez J, Hatting A. Contribución al estudio del tratamiento del raquitismo por el golpe vitamínico. Rev chil pediatr. 1941; 12(3): 214-225.

9. Así como el 18 de septiembre se conmemora la independencia política de Chile, la marca registrada 18 significa para nuestros productos la independencia industrial de Chile. En: Almanaque Daube. 1923.

10. Revista Químico Farmacéutica. 1943 oct; $\mathrm{I}(8): 19$.

11. Revista Químico Farmacéutica. 1945 feb; III(25): 19.

12. Almanaque 18.1947.

13. Alcances sobre la creación de la carrera de Química y Farmacia en Valparaíso. Revista Colegio Químico Farmacéutico. 1972; XXIX(298).

14. El Instituto de Química de la U. de Chile de Valparaíso. Revista Colegio Químico Farmacéutico. 1972; XXIX(298).

15. Alviña T. Crónica de la Escuela de Química y Farmacia Universidad de Valparaiso: 1972-1989. Santiago: Academia de Ciencias Farmacéuticas de Chile; 1990. 